\title{
Exercise training and rehabilitation of the brain in Parkinson's disease
}

\author{
Muhammed Al-Jarrah \\ Department of Rehabilitation Sciences. Faculty of Applied Medical Sciences. Jordan University of Science and Technology.22110, Irbid \\ - Jordan
}

Email address:

jarrahm@just.edu.jo (M. Al-Jarrah)

To cite this article:

Muhammed Al-Jarrah. Exercise Training and Rehabilitation of the Brain in Parkinson's Disease. Clinical Medicine Research. Vol. 2, No. 2, 2013, pp. 11-17. doi: 10.11648/j.cmr.20130202.12

\begin{abstract}
Parkinson's disease (PD) is one of the most chronic progressive neurodegenerative diseases that is clinically manifested by of cardinal motor symptoms including, tremor and rigidity. The known cause of PD is the loss of dopaminergic neurons in substantia nigra in the brain. There are motor and non motor features of this disease with heterogenic complaints. The main treatment available for PD is levodopa as dopamine replacement therapy. However, after few years of treatment, PD patients experience levodopa-resistant symptoms. Other neurosurgical procedures for the treatment of PD have become a widely performed. These surgical procedures stimulate the dopaminergic neurons to produce more dopamine, but won't halt the progression of degeneration of these cells. Over the last years, many studies focused on the effect of physical therapy on PD, most of these studies have investigated the rehabilitation effects on musculoskeletal system, like gait, balance, and strength. Other studies focused on the effect of physiotherapy on non motor feature in PD, like quality of life. However, there is limited information about the beneficial impact of exercise on the brain of PD patients. In this review, we provide a brief review of the literature on exercise effects on the brain of PD. The present review was designed to gain more insight into the mechanism of improvement in PD patients with exercise and to answer in part the question of how exercise training rehabilitates the brain in PD patients.
\end{abstract}

Keywords: Exercise, Brain, Parkinson's disease

\section{Introduction}

Parkinson's disease (PD), one of the most common causes of disability among the elderly [1,2] is a movement disorder characterized by akinesia (the inability to initiate movement), tremors and muscular rigidity. At the physiological level, PD is caused by a deficiency of dopamine, which is a neurotransmitter that sends messages between nerve cells in the mammalian brain. First described by James Parkinson, PD has been lately defined as a collection of symptoms rather than one distinct clinical picture ${ }^{[3]}$.Thus, the diagnosis of PD can be difficult in the early stages of the disease.

PD progression is highly variable. Early PD symptoms typically include forward flexion of the neck, hips, knees, and elbows. Postural abnormalities greatly reduce the patients' ability to be ambulatory and places them at high risk for injury and falls $[4,5,6]$. Postural instability is a common and serious problem in PD [7]. Because of rigidity and postural changes, patients also develop a shuffling quality to their gait and use small steps. Decreased speed and stride length are also main disturbances of gait in PD [8].

The exact benefits of exercise as a treatment for motor disability are not clear, yet as there are a small number of studies that support the concept. In one of the first reports in the literature on physical therapy for PD in 1961, Doshay compared $100 \mathrm{PD}$ patients who did not receive any physical therapy with 100 PD patients who had intensive physical therapy for several years. He found, on average, less severely disabled patients in the exercising group when measuring both early and late time points over several years [9]. So, what would be the reason behind this theory considering the brain?

\subsection{Mechanisms of Effects of Exercise on the Brain of PD Patients}

In three large cohort studies, it was reported that moderate to vigorous exercise habits in midlife significantly reduced the risk of later-developing PD [10,11,12]. However, the mechanisms responsible for these changes are not 
well investigated. Here, the possible mechanisms of action of exercise will be discussed and reviewed.

\subsection{Neurotrophic Factors and Impact of Exercise in PD}

Angiogenesis is the formation of new blood vessels. This process does not occur without stimulation. In PD, studies have suggested that vascular endothelial growth factor (VEGF) signaling may result in neuroprotective effects through the enhancement of dopaminergic neuron survival [11]. So an increased VEGF level in the affected areas in the brain in PD might have a potential therapeutic effect $[12,13]$. Beyond the neuroprotective role, Yasuhara reported that VEGF may also play a part in neurogenesis itself [14]. Using a rat model of PD they tested the neuroprotective capacity of VEGF. They found that rats receiving a continuous infusion of VEGF into the brain displayed a significant decrease in amphetamine-induced rotational behavior and a significant preservation of tyrosine hydroxylase-positive neurons and fibers compared with control animals. In our previous studies on the brain of Parkinsonian mouse, we reported increases angiogenesis on the striatum of exercised PD mice compared to sedentary PD ones [15]. These results were addressed in the way that neuroprotection role of VEGF is mediated indirectly by promoting angiogenesis in exercise.

In $\mathrm{PD}$, blood flow to the brain has a functional importance not only for promoting the survival of the affected dopaminergic neurons by providing more oxygen and nutrients, but it may also help with efficient drug delivery to the affected areas. This is especially important for levodopa since a large proportion of the drug does not reach the brain, because it is rapidly converted to dopamine [16]. Increased blood flow to the affected areas of the brain would definitely help with more efficient drug delivery.

Another possible mechanism of improvement of PD with exercise is through a substance released by cells called glia derived neurotrophic factors (GDNF). GDNF is a neurotrophic factor that enhances survival and morphological differentiation of dopaminergic neurons and increases their dopamine uptake [17,18]. Despite extensive study, the mechanism of action of GDNF in cell and animal models of PD is far from fully elucidated. In dopaminergic neurons, GDNF binds to the a1-subtype of GDNF family cell surface receptors, the receptors signal through receptor tyrosine kinase and can thereby phosphorylate different intracellular targets in the cell. [19].

GDNF has significant actions in promoting neuronal survival in the central nervous system, taken into consideration that GDNF can exert its neurotrophic effects at the level of cell bodies in the substantia nigra and in the axon terminals in the striatum ${ }^{[20,21]}$. Compared to other growth factors, GDNF's ability to ensure survival of dopaminergic neurons is superior, and considered as a treatment option for PD [21,22]. When directly compared to brain-derived neurotrophic factor (BDNF), GDNF corrected behavioral deficits and protected nigrostriatal dopaminergic neurons better than BDNF. In addition, expression of both neuro- trophic factors was no more effective than expression of GDNF alone [23].

In Gash's laboratory monkeys with PD-like symptoms showed significant functional improvements in bradykinesia, rigidity and postural instability with GDNF infusion. In the affected area, GDNF increased dopamine levels in the midbrain and globus pallidus [24]. Another study by Zheng et al. showed that GDNF significantly increased the number of neurons and the dopamine levels in the brain, with a subsequent behavioral recovery in Parkinsonian rats. These findings demonstrate that gene transfer of GDNF is efficacious even in the late stages of PD in rats [25,26]. These results were confirmed recently by $\mathrm{Du}$ and his colleagues who showed that GDNF gene therapy can protect the nigral DA neurons from degeneration. [27]

In human studies and based on the results of some of the animal studies, clinical trials using GDNF followed the same trend. Gill et al delivered GDNF directly into the putamen of five Parkinson's patients and their research results showed significant improvement of dopamine uptake and storage. The study also showed significant improvement with the Unified Parkinson's Disease Rating Scale (UPDRS) motor score, and ADLs of those patients [28]. Another study by Love et al provided the first neuropathological evidence that infusion of GDNF into the brain of patients with advance PD caused sprouting of dopaminergic fibers and a marked increase in the dopamineproducing nerve fibers similar to that seen in animal models [29]. Considering the long term effect of this GDNF treatment approach, a study by Patel, showed that following two years of injection of GDNF, PD patients did not show any serious side effects, and there was significant improvement in their off-medication status, QoL measures, UPDRS, and ADLs [30]. Other studies with 6 month infusion periods have shown similar results [31]. Thus, the human studies support the role of GDNF in improving neuronal function and clinical outcomes for patients with PD. Similarly, walking distances as indices of physical activity were associated with better preservation of gray matter/cortical volumes when followed up 9 years later [32] .These encouraging finding qualifies GDNF as the most interesting candidate factor to date for treating PD. As exercise training increased the level these neurotrophic factors, it could then explain in part this beneficial effect on the brain of PD patients. Moreover, treadmill exercise training was reported as potential factor for modulating neurochemical status in the striatum of rats, and promote neuroprotective role possibly by improving the oxidative stress factors $[33,34]$, so there is also a positive role of exercise on the status of neurotransmitter in the brain of PD rats. A study by Klaissle reported that exercise regulate the generation of neural precursors in substantia nigra of rat with induced Parkinsonism (Klaissle, 2012 Oct 31;13:132). PD from another aspect is classified as brain damage related disease. In regard to those harmful factors and brain damage markers, we reported positive effect of exercise in limiting the effect of those damaging factors in the striatum 
of Parkinsonian mouse and we showed improvement in the production of glial derived proteins with exercise [36]. In consistent with our findings, Dutra recently reported that exercise led to restored striatal glial fibrillary acidic protein (GFAP) expression in the striatum of Parkinsonian rat [37]. In early PD in human clinical trials, exercise also showed improvement in the motor performance and corticomotor excitability [38]. Even when patient will go to surgery for deep brain stimulation, positron emission tomography studies showed that, exercise still have recognized effect and continue to be a major part of the treatment, as it causes significant dopamine release in the ventromedial striatum and in the putamen [39].

\subsection{Neuroinflamatory Components, Oxidative Damage and Exercise in PD}

In regard to inflammatory processes in human body, the inflammatory component of PD is having significant attention among researchers now. Chronic inflammation is a major risk factor underlying aging and age-related diseases, like PD [40], as well as a central component to the notion of immunosenescence. On the other hand, previous studies by Ferrari and Tarelli showed how systemic inflammation may impact central inflammation and dopaminergic cell death [41]. These theories raise the possibility that peripheral inflammation could be a contributing factor in PD development. Moreover, studies in the animal models of PD by Machado supported the theory of neuroinflamation in PD [42].

The theory of neuroinflamation in PD came from the idea of microglial activation. Microglial cells can be considered as the resident macrophages of the brain and major immune cells in the CNS. The presence of microglial activation has been reported in Substantia nigra in PD in both human and animal studies $[43,44]$. Neurons in the SN have been shown to be particularly susceptible to microglialmediated toxicity in vitro and in vivo [45]. Also, in another large epidemiological study conducted by Honglei in to test the relationship between inflammatory markers and diagnosis of PD in human blood samples, they found that higher level of interleukin-6 was associated with a greater risk of PD [46]. Moreover, in animal models of PD, antiinflammatory intervention protocols were reported to be neuroprotective $[47,48,49,50]$.

Oxidative stress is also thought to promote tissue damage in PD [51]. Generally, oxidative stress is generated by the inability to detoxify or to repair the resulting damage caused by the generation of reactive oxygen species. Mitochondrial damage as a result of oxidative stress has been reported in human PD studies [52]. Other oxidative damage ways has been reported through excitotoxicity, and nitric oxide toxicity [53]. On the other hand, the blocking of angiotensin receptor 1 on oxidative stress or/and microglial activation have recently been demonstrated in the MPTP model of PD to have a neuroprotective effects [54], Here, the main goal of this review is not to clari- fy deeply the Neuroinflamatory process itself in PD, or investigating the oxidative damage in $\mathrm{PD}$, but rather to review the beneficial effect of exercises on those factors.

In $\mathrm{PD}$, there is alteration in the neuroimmunological function [55]. Recently in Sweden, Archer reported that physical activity diminishes inflammation and elevates agents and factors involved in immunomodulatory function [55], he also found that different types of exercise attenuates or reverses the performance deficits observed in neurocognitive tasks. The effect of physical activity has been also reported by $\mathrm{Wu}$ et al to have a neuroprotective role on dopaminergic neurons (DA) against inflammationinduced insults, this study also showed that four weeks of running exercise before an intraperitoneal lipopolysaccharide ((LPS) injection; a drug that can induce degeneration in the SN and reduced the level of dopamine in the striatum) treatment completely prevented the LPS-induced loss of DA neurons [56]. These results came in agreement with the findings of Johnston and his colleagues who found that human interleukin-10 Gene transfer was protective in a Rat Model of PD [57]. Even when the case of PD will go to deep brain stimulation (DBS) surgery, Nozaki and his colleagues recently found significant dopamine release in the ventromedial striatum by STN-DBS during exercise, which indicates that dopaminergic activation process occurs in the motivational circuit during action [58].

Concerning the effect of physical activity and oxidative damage in PD, recent studies also showed that exercise protects the mitochondrial proteins damage in $\mathrm{PD}{ }^{[38]}$. These results were also supported by Patki and Lau who showed the positive impact of exercise on mitochondrial function as the function of mitochondria is essential for preventing the process of neurodegeneration in PD [59]. In human studies, resistance exercise training also showed to be associated with reduced oxidative stress in subjects with PD. This reduction can be explained by increase endogenous antioxidant protection role and decrease the production of reactive oxygen and nitrogen species [60].

\subsection{Exercise Effect on PD Medications}

To date there is no cure for PD. Levodopa as golden standard medication for PD might results in Levodopa resistance or levodopa toxicity after 5-7 years of treatment. The dose has important role on the efficacy of the drug as we need to reduce the drug option in PD treatment by replacing this approach with safe and feasible way of treatment for PD. In this regard, studies showed that intensive rehabilitation program lead to reduction in daily medication dosage in exercised treated PD patients, while it was increased significantly in control patients [61]. Recent studies from Cleveland Clinic showed that forced exercise of lower extremities reduced the motor symptoms in PD, moreover, this study also showed that the activation of the brain region in $\mathrm{PD}$ using functional magnetic resonance imaging by exercise has the same pattern of activation after medication [62], so both exercise and medication might use 
the same pathway to reduce the progression of PD. In our previous report, we showed increase production of dopamine in chronic Parkinsonian mice, although that increase was not significant, but it is of great clinical value as exercise wont only decrease the worsening of the disease but also able to increase the production of dopamine [63]. In agreement with our previous results, Muller and Muhlack concluded that endurance exercise induced increase in endogenous dopamine synthesis, which is indicated by beneficial effect on scored motor performance in patients with PD [64]. These excited results placed exercise therapy approach as alternative that has almost the same impact of medications in PD.

\subsection{Exercise Improves Cognition and Memory in PD}

Large epidemiological studies showed that physical activity is associated with decreased risk of cognitive impairment in Alzheimer's disease; these studies showed that people who exercise routinely are less prone to have Alzheimer's disease late in their life [65]. With aging, there is a physiological decline in brain function [66]. The hippocampus will shrink; often result in memory decline and impairment [67]. One of the major complications in PD as age associated diseases cognitive impairment [68]. Hippocampus is the structure of importance in memory issues. Here, there are studies which showed that aerobic exercise training is effective at reversing hippocampal volume loss in late adulthood as exercise increases the size of the anterior hippocampus. Increasing the size of hippocampus will consequently improve the memory in PD patients [69]. Other studies in this regard also showed that physical activity spares gray matter volume in late adulthood, so Physical activity is associated with reduced risk of cognitive impairment [70,71]. Another study by Laura and colleagues to examine whether exercise is beneficial for people with dementia and related cognitive impairments, they found that exercise training increases cognitive function, and positive behavior in people with dementia and related cognitive impairments [72]. There is more than one hypothesis behind the fact that physical activity reduces the risk of reduced cognition with aging and with age related diseases. One of these molecular basis hypothesis was reported by Gomeza and colleagues, who found that fibroblast growth factor messenger RNA, and astrocytes density were increased in hippocampus and cerebellum of trained rats [73]. Other researchers have reported increased synaptic plasticity with exercise training through its effect of hippocampal BDNF [74]. Here it would be important to point to the fact that BDNF influences the development of patterned connections growth and complexity of dendrites in the brain [75]. In our previous research on mouse model with chronic Parkinsonism, we reported increased expression of glial fibrillary acidic protein in the striatum [36] and increased neurogenesis [15]. In agreement with our results, Saur and colleagues recently reported that physical exercise increases GFAP expression and induces morphological changes in hippocampal astrocytes [76]. These results raised the importance of physical activity on brain plasticity, and activation and stabilization of neuronal synapses in the brain.

In conclusion, exercise training can be considered as safe and feasible approach to rehabilitate PD. The effect of exercise is way beyond the improvement of quality of life, increased balance and muscle strength, but rather than that exercise has good ability to rehabilitate the brain in PD.

\section{Acknowledgment}

The author would like to thank the Deanship of Research at Jordan University of Science and Technology for their support.

\section{References}

[1] Lima LO, Rodrigues-de-Paula F. Muscular power training: a new perspective in physical therapy approach of Parkinson's disease. Rev Bras Fisioter. 2012,16(2):173-4.

[2] Fetsko LA, Xu R, Wang Y. Effects of age and dopamine $\mathrm{D} 2 \mathrm{~L}$ receptor-deficiency on motor and learning functions.Neurobiol Aging 2005;26(4):521-30.

[3] Ghosh B, Mishra A, Sengupta P. Is Parkinson's disease a homogeneous disorder--what is the burden of Parkinson's disease in India. J Indian Med Assoc. 2005;103(3):146, 148, 150 passim.

[4] Balash Y, Peretz C, Leibovich G, Herman T, Hausdorff JM, Giladi N. Falls in outpatients with Parkinson's disease Frequency, impact and identifying factors. J Neurol. 2005;1310.

[5] Contreras A, Grandas F. Risk factors for freezing of gait in Parkinson's disease. J Neurol Sci. 2012 Sep 15;320(12):66-71

[6] Bryant MS, Rintala DH, Hou JG, et al. The relation of falls to fatigue, depression and daytime sleepiness in Parkinson's disease. Eur Neurol. 2012;67(6):326-30.

[7] Agari T, Date I. Spinal cord stimulation for the treatment of abnormal posture and gait disorder in patients with Parkinson's disease. Neurol Med Chir (Tokyo). 2012;52(7):470-4.

[8] Pohl M, Rockstroh G, Ruckriem S, Mrass G, Mehrholz J. Immediate effects of speed-dependent treadmill training on gait parameters in early Parkinson's disease. Arch Phys Med Rehabil 2003;84(12):1760-6.

[9] Doshay LJ. Current concepts in therapy. Treatment of Parkinson's disease. 1. Medicinal therapy. 2. N Engl J Med. 1961;264:1097-9.

[10] Chen H, Zhang SM, Schwarzschild MA, Hernan MA, Ascherio A. Physical activity and the risk of Parkinson disease. Neurology 2005;64:664-669.

[11] Thacker EL, Chen H, Patel AV, et al. Recreational physical activity and risk of Parkinson's disease.Mov Disord 2008;23:69-74. [12] Xu Q, Park Y, Huang X, et al. Physical activities and future risk of Parkinson disease. Neurology2010;75:341-348. 
[12] Pitzer MR, Sortwell CE, Daley BF, McGuire SO, Marchionini D, Fleming M, Collier TJ. 2003. Angiogenic and neurotrophic effects of vascular endothelial growth factor (VEGF165): studies of grafted and cultured embryonic ventral mesencephalic cells. ExpNeurol 182(2):435-45.

[13] Silverman WF, Krum JM, Mani N, Rosenstein JM. 1999. Vascular, glial and neuronal effects of vascular endothelial growth factor in mesencephalic explant cultures. Neuroscience 90(4):1529-41.

[14] Yasuhara T, Shingo T, Date I. 2004a. The potential role of vascular endothelial growth factor in the central nervous system. Rev Neurosci 15(4):293-307.

[15] Al-Jarrah M, Jamous M, Al Zailaey K, et al. Endurance exercise training promotes angiogenesis in the brain of chronic/progressive mouse model of Parkinson's Disease. NeuroRehabilitation. 2010;26(4):369-73.

[16] Da Prada M, Kettler R, Keller HH, Burkard WP, MuggliManiglio D, Haefely WE. 1989. Neurochemical profile of moclobemide, a short-acting and reversible inhibitor of monoamine oxidase type A. J PharmacolExpTher 248(1):40014.

[17] Barroso-Chinea P, Cruz-Muros I, Aymerich MS, RodriguezDiaz M, Afonso-Oramas D, Lanciego JL, GonzalezHernandez T. 2005.Striatal expression of GDNF and differential vulnerability of midbrain dopaminergic cells.Eur $\mathrm{J}$ Neurosci 21(7):1815-27.

[18] Campos FL, Cristovão AC, Rocha SM, et al. GDNF Contributes to Oestrogen-Mediated Protection of Midbrain Dopaminergic Neurones. J Neuroendocrinol. 2012 Nov;24(11):1386-97

[19] Airaksinen MS, Saarma M. 2002. The GDNF family: signalling, biological functions and therapeutic value. Nat Rev Neurosci 3(5):383-94.

[20] Anastasía A, Wojnacki J, de Erausquin GA, et al. Glial cellline derived neurotrophic factor is essential for electroconvulsive shock-induced neuroprotection in an animal model of Parkinson's disease. Neuroscience. 2011,10,(195):100-11.

[21] Bowenkamp KE, David D, Lapchak PL, et al. 6hydroxydopamine induces the loss of the dopaminergic phenotype in substantianigra neurons of the rat. A possible mechanism for restoration of the nigrostriatal circuit mediated by glial cell line-derived neurotrophic factor.Exp Brain Res. 1996,111(1):1-7.

[22] Duarte EP, Curcio M, Canzoniero LM, et al. Neuroprotection by GDNF in the ischemic brain. Growth Factors. 2012,30(4):242-57.

[23] Sun M, Kong L, Wang X, Lu XG, Gao Q, Geller AI. Comparison of the capability of GDNF, BDNF, or both, to protect nigrostriatal neurons in a rat model of Parkinson's disease. Brain Res. 2005.1052(2):119-29.

[24] Gash DM, Zhang Z, Ovadia A, CassWA, Yi A, Simmerman L, Russell D, Martin D, Lapchak PA, Collins F and others. Functional recovery in parkinsonian monkeys treated with GDNF. Nature. 1996,380(6571):252-5.

[25] Zheng JS, Tang LL, Zheng SS, Zhan RY, Zhou YQ, Goudreau J, Kaufman D, Chen AF. Delayed gene therapy of glial cell line-derived neurotrophic factor is efficacious in a rat model of Parkinson's disease. Brain Res Mol Brain Res.
2005. 134(1):155-61.

[26] Grondin R, Zhang Z, Yi A, Cass WA, Maswood N, Andersen AH, Elsberry DD, Klein MC, Gerhardt GA, Gash DM. Chronic, controlled GDNF infusion promotes structural and functional recovery in advanced parkinsonian monkeys. Brain. 2002. 125(10):2191-201.

[27] Du Y, Zhang X, Tao Q, Chen S, Le W. Adeno-Associated Virus Type 2 Vector-Mediated Glial Cell Line-Derived Neurotrophic Factor Gene Transfer Induces Neuroprotection and Neuroregeneration in a Ubiquitin-Proteasome System Impairment Animal Model ofParkinson's Disease. Neurodegener Dis. 2012 (24): 1-16

[28] Gill SS, Patel NK, Hotton GR, O'Sullivan K, McCarter R, Bunnage M, Brooks DJ, Svendsen CN, Heywood P. Direct brain infusion of glial cell line-derived neurotrophic factor in Parkinson disease. Nat Med. 2003, 9(5):589-95.

[29] Love S, Plaha P, Patel NK, Hotton GR, Brooks DJ, Gill SS. Glial cell line-derived neurotrophic factor induces neuronal sprouting in human brain. Nat Med 2005, 11(7):703-4.

[30] Patel NK, Bunnage M, Plaha P, Svendsen CN, Heywood P, Gill SS. Intraputamenal infusion of glial cell line-derived neurotrophic factor in PD: a two-year outcome study. Ann Neurol. 2005, 57(2):298-302.

[31] Slevin JT, Gerhardt GA, Smith CD, Gash DM, Kryscio R, Young B. Improvement of bilateral motor functions in patients with Parkinson disease through the unilateral intraputaminal infusion of glial cell line-derived neurotrophic factor. J Neurosurg 2005,102(2):216-22.

[32] Erickson KI, Raji CA, Lopez OL, et al. Physical activity predicts gray matter volume in late adulthood: The Cardiovascular Health Study. Neurology 2010;75:1415-1422.

[33] Meeusen, R; Smolders, I; Sarre, S; et al. Endurance training effects on neurotransmitter release in rat striatum: an in vivo microdialysis study. Acta Physiologica Scandinavica vol. 1997, 159 (4): 335-341

[34] Tuon T, Valvassori SS, Lopes-Borges J, et al. Physical training exerts neuroprotective effects in the regulation of neurochemical factors in an animal model of Parkinson's disease. Neuroscience. $2012 ; 227: 305-12$.

[35] Philipp Klaissle, Anne Lesemann, Petra Huehnchen, Andreas Hermann, Alexander Storch, Barbara Steiner. Physical activity and environmental enrichment regulate the generation of neural precursors in the adult mouse substantia nigra in a dopamine-dependent manner. BMC Neurosci. 2012; 13: 132 .

[36] Al-Jarrah MD, Jamous M. Effect of endurance exercise training on the expression of GFAP, S100B, and NSE in the striatum of chronic/progressive mouse model of Parkinson's disease. NeuroRehabilitation. 2011;28(4):359-63.

[37] Dutra MF, Jaeger M, Ilha J, et al. Exercise improves motor deficits and alters striatal GFAP expression in a 6-OHDAinduced rat model of Parkinson's disease. Neurol Sci. 2012;33(5):1137-44.

[38] Fisher BE, Wu AD, Salem GJ, et al. The effect of exercise training in improving motor performance and corticomotor excitability in people with early Parkinson's disease. Arch Phys Med Rehabil. 2008;89(7):1221-9. 
[39] Nozaki T, Sugiyama K, Yagi S, et al. Effect of subthalamic nucleus stimulation during exercise on the mesolimbocortical dopaminergic region inParkinson's disease: a positron emission tomography study. J Cereb Blood Flow Metab. 2012 Dec 5.

[40] Malú G. Tansey and Matthew S. Goldberg. Neuroinflammation in Parkinson's disease: its role in neuronal death and implications for therapeutic intervention. Neurobiol Dis. 2010; 37(3): 510-518

[41] Carina C. Ferrari, Rodolfo Tarelli. Parkinson's Disease and Systemic Inflammation. Parkinsons Dis. 2011; 2011

[42] A.Machado, A. J. Herrera, J. L. Venero, M. Santiago, R. M. De Pablos, R. F. Villar'an, A. M. Espinosa-Oliva, S. Arg"uelles, M. Sarmiento. M. J. Delgado-Cort'es, R.Mauri no, and J. Cano. Peripheral inflammation increases the damage in animal models of nigrostriatal dopaminergic neurodegeneration: Possible implication in Parkinson's disease incidence. Parkinson's Disease, 2011, 2011:1-10.

[43] Perry VH, Nicoll JA, Holmes C. Microglia in neurodegenerative disease. Nat Rev Neurol. 2010 ;6(4):193-201.

[44] McGeer PL, Itagaki S, Boyes BE, McGeer EG. Reactive microglias are positive for HLA-DR in the substantia nigra of Parkinson's and Alzheimer's disease brains. Neurology. $1988 ; 38(8): 1285-91$.

[45] W. G. Kim, R. P. Mohney, B. Wilson, G. H. Jeohn, B. Liu, and J. S. Hong, "Regional difference in susceptibility to lipopolysaccharide-induced neurotoxicity in the rat brain: role of microglia," The Journal of Neuroscience, vol. 20, no. 16, pp. 6309-6316, 2000.

[46] Honglei Chen, Eilis J. O’Reilly, Michael A. Schwarzschild, and Alberto Ascherio Peripheral Inflammatory Biomarkers and Risk of Parkinson's Disease. Am J Epidemiol 2008;167:90-95.

[47] Y. He, S. Appel, and W. Le, "Minocycline inhibits microglial activation and protects nigral cells after 6hydroxydopamine injection into mouse striatum," Brain Research, vol. 909, no. 1-2, pp. 187-193, 2001.

[48] Andreas Hald, Julie Lotharius. Oxidative stress and inflammation in Parkinson's disease: is there a causal link? Experimental Neurology, Volume 193, Issue 2, June 2005, Pages 279-290.

[49] R. Sánchez-Pernaute, A. Ferree, O. Cooper, M. Yu, A. L. Brownell, and O. Isacson, "Selective COX-2 inhibition prevents progressive dopamine neuron degeneration in a rat model of Parkinson's disease," Journal of Neuroinflammation, vol. 1, article 6, 2004.

[50] D. C. Wu, V. Jackson-Lewis, M. Vila et al., "Blockade of microglial activation is neuroprotective in the 1-methyl-4phenyl-1,2,3,6-tetrahydropyridine mouse model of Parkinson disease," The Journal of Neuroscience, vol. 22, no. 5, pp. 1763-1771, 2002.

[51] De-Hyung Lee, Ralf Gold and Ralf A. Linker Mechanisms of Oxidative Damage in Multiple Sclerosis and Neurodegenerative Diseases: Therapeutic Modulation via Fumaric Acid Esters. Int J Mol Sci, 2012;13(9):11783-803

[52] Zafar I. Alam, Susan E. Daniel, Andrew J. Lees, Davjd C. Marsden, Peter Jenner, and Barry Halliwell. A Generalised Increase in Protein Carbonyls in the Brain in Parkinson's but Not Incidental Lewy Body Disease. J. Neurochem., Vol. 69, No. 3, 1997.

[53] Jenner P. Dopamine agonists, receptor selectivity and dyskinesia induction in Parkinson's disease. Curr Opin Neurol, 2003 Dec;16 Suppl 1:S3-7.

[54] Juan M. SAAVEDRA, Enrique SÁNCHEZ-LEMUS, Julius BENICKY. Blockade of brain angiotensin II AT1 receptors ameliorates stress, anxiety, brain inflammation and ischemia: Therapeutic implications. Psychoneuroendocrinology. 2011 January ; 36(1): 1-18.

[55] Archer T, Fredriksson A, Schütz E, Kostrzewa RM. Influence of physical exercise on neuroimmunological functioning and health: aging and stress. Neurotox Res. 2011 Jul;20(1):69-83.

[56] Wu SY, Wang TF, Yu L, Jen CJ, Chuang JI, Wu FS, Wu CW, Kuo YM. Running exercise protects the substantia nigra dopaminergic neurons against inflammation-induced degeneration via the activation of BDNF signaling pathway. Brain Behav Immun. 2011 Jan;25(1):135-46.

[57] Johnston LC, Su X, Maguire-Zeiss K, Horovitz K, Ankoudinova I, Guschin D, Hadaczek P, Federoff HJ, Bankiewicz $\mathrm{K}$, Forsayeth J. Human interleukin-10 gene transfer is protective in a rat model of Parkinson's disease. Mol Ther. 2008 August ; 16(8): 1392-1399.

[58] Takao Nozaki, Kenji Sugiyama, Shunsuke Yagi, Etsuji Yoshikawa,Toshihiko Kanno, Tetsuya Asakawa, Tae Ito, Tatsuhiro Terada, Hiroki Namba and Yasuomi Ouch .Effect of subthalamic nucleus stimulation during exercise on the mesolimbocortical dopaminergic region in Parkinson's disease: a positron emission tomography study. J cereb Blood Flow 2012 Dec :183.

[59] Patki G, Lau YS. Impact of exercise on mitochondrial transcription factor expression and damage in the striatum of a chronic mouse model of Parkinson's disease. Neurosci Lett. 2011 Nov 21;505(3):268-72.

[60] Bloomer RJ, Schilling BK, Karlage RE, Ledoux MS, Pfeiffer RF, Callegari J. Effect of resistance training on blood oxidative stress in Parkinson disease. med sci sports exerc, 2008 aug;40 (8) 1385-9.

[61] Frazzitta G, Bertotti G, Riboldazzi G, Turla M, Uccellini D, Boveri N, Guaglio G, Perini M, Comi C, Balbi P, Maestri R. Effectiveness of intensive inpatient rehabilitation treatment on disease progression in parkinsonian patients: a randomized controlled trial with 1-year follow-up. Neurorehabil Neural Repair, 2011: 144-150.

[62] Beall E, Lowe M, Alberts JL, Frankemolle AM, Thota AK, Shah C, Phillips MD.The Effect of Forced-Exercise Therapy for Parkinson's Disease on Motor Cortex Functional Connectivity. Brain Connect. 2013 Jan 14.

[63] Al-Jarrah M, Pothakos K, Novikova L, Smirnova IV, Kurz MJ, Stehno-Bittel L, Lau YS. Endurance exercise promotes cardiorespiratory rehabilitation without neurorestoration in the chronic mouse model of parkinsonism with severe neurodegeneration. Neuroscience. 2007 Oct 12;149(1):28-37.

[64] Thomas Mu"ller and Siegfried Muhlack. Effect of exercise on reactivity and motor behaviour in patients with Parkinson's disease. J Neurol Neurosurg Psychiatry 2010;81:747e753 
[65] Arthur F. Kramer, Kirk I. Erickson, and Stanley J. Colcombe. Exercise, cognition, and the aging brain. J Appl Physiol, 2006, 101: 1237-1242.

[66] Keleshian VL, Modi HR, Rapoport SI, Rao JS . Aging is associated with altered inflammatory, arachidonic acid cascade and synaptic markers, influenced by epigenetic modifications, in the human frontal cortex. J Neurochem. 2013 Jan 22 .

[67] K.I. Erickson, C.A. Raji, O.L. Lopez, J.T. Becker, C. Rosano, A.B. Newman, H.M. Gach, P.M. Thompson, A.J. Ho, L.H. Kuller. Physical activity predicts gray matter volume in late adulthood: the Cardiovascular Health Study. Neurology 75 October 19, 2010.

[68] Weintraub D, Doshi J, Koka D, Davatzikos C, Siderowf AD, Duda JE, Wolk DA, Moberg PJ, Xie SX, Clark CM. Neurodegeneration across stages of cognitive decline in Parkinson disease. Arch Neurol. 2011 December ; 68(12): 1562-1568.

[69] Kirk I. Erickson, Michelle W. Voss, Ruchika Shaurya Prakash, Chandramallika Basak, Amanda Szabo,Laura Chaddock, Jennifer S. Kim, Susie Heo, Heloisa Alves, Siobhan M. White, Thomas R. Wojcicki,Emily Mailey, Victoria J. Vieira, Stephen A. Martin, Brandt D. Pence, Jeffrey A. Woods, Edward McAuley and Arthur F. Kramer. Exercise training increases size of hippocampus and improves memory. PNAS , 2011. 108, 7, 3017-3022.

[70] Michelle W. Voss, Lindsay S. Nagamatsu, Teresa LiuAmbrose, Arthur F. Kramer. Exercise, brain, and cognition across the life span. J Appl Physiol. 2011 November; 111(5):
$1505-1513$.

[71] K.I. Erickson, C.A. Raji, O.L. Lopez, J.T. Becker, C. Rosano, A.B. Newman, H.M. Gach, P.M. Thompson, A.J. Ho, L.H. Kuller. Physical activity predicts gray matter volume in late adulthood. Neurology. 2010 October 19; 75(16): $1415-1422$.

[72] Laura D. Baker, Laura L. Frank, Karen Foster-Schubert, Pattie S. Green, Charles W. Wilkinson, Anne McTiernan, Stephen R. Plymate, Mark A. Fishel, G. Stennis Watson, Brenna A. Cholerton, Glen E.Duncan, Pankaj D. Mehta, and Suzanne Craft. Effects of Aerobic Exercise on Mild Cognitive Impairment: A Controlled Trial. Arch Neurol. 2010 January ; 67(1): 71-79.

[73] F Gomeza-Pinilla. V So, J Pl Kisslak. Spatial learning induces neurotrophin receptor and synapsin I in the hippocampus. Brain Res. 2001:13-19.

[74] Shoshanna Vaynman' Zhe Ying' Fernando Gomez-Pinilla. Hippocampal BDNF mediates the efficacy of exercise on synaptic plasticity and cognition Eur J NeuroSci .2004 Nov;20(10):2580-90.

[75] Yamada K, Mizuno M, Nabeshima T. Role for brain-derived neurotrophic factor in learning and memory. Life Sci, 2002 Jan 4;70(7):735-44.

[76] Saur L, Baptista PP, de Senna PN, Paim MF, Nascimento PD, Ilha J, Bagatini PB, Achaval M, Xavier LL. Physical exercise increases GFAP expression and induces morphological changes in hippocampal astrocytes. Brain Struct Funct. 2013 Jan 4. 